\title{
$T$-SPACES BY THE GOTTLIEB GROUPS AND DUALITY
}

\author{
MOO HA WOO and YEON SOO YOON
}

(Received 26 April 1991; revised 1 February 1993)

Communicated by J. H. Rubinstein

\begin{abstract}
It is shown that all the generalized Whitehead products vanish in $X$ and all the components of $X^{\Sigma A}$ have the same homotopy type when $X$ is a $T$-space. It is also shown that any $T$-space is a $G$-space. The dual spaces of $T$-spaces are introduced and studied.
\end{abstract}

1991 Mathematics subject classification (Amer. Math. Soc.): 55P45, 55P35.

Keywords and phrases: cyclic maps, cocyclic maps, $T$-spaces, co- $T$-spaces.

\section{Introduction}

A based map $f: A \rightarrow X$ is called cyclic [13] if there exists a map $F: X \times A \rightarrow X$ such that $F j \sim \nabla(1 \vee f)$, where $j: X \vee A \rightarrow X \times A$ is the inclusion and $\nabla: X \vee X \rightarrow X$ is the folding map. The Gottlieb set, denoted $G(A, X)$, is the set of all homotopy classes of cyclic maps from $A$ to $X$. The concept of cyclic maps was first introduced and studied by Gottlieb [5] and Varadarajan [13]. Gottlieb [6] introduced and studied the evaluation subgroups $G_{m}(X)$ of $\pi_{m}(X) . G_{m}(X)$ is defined to be the set of all homotopy classes of cyclic maps from $S^{m}$ to $X$. A space $X$ satisfying $G_{m}(X)=$ $\pi_{m}(X)$ for all $m$ is called a $G$-space. It is known [6] that any $H$-space is a $G$-space. On the other hand, a based map $f: X \rightarrow A$ is called cocyclic [13] if there exists a map $\phi: X \rightarrow X \vee A$ such that $j \phi \sim(1 \times f) \Delta$, where $j: X \vee A \rightarrow X \times A$ is the inclusion and $\Delta: X \rightarrow X \times X$ is the diagonal map. The dual Gottlieb set, denoted $D G(X, A)$, is the set of all homotopy classes of cocyclic maps from $X$ to $A$. Haslam [7] introduced and studied the coevaluation subgroups $G^{m}(X)$ of $H^{m}(X)$. $G^{m}(X)$ is defined to be the set of all homotopy classes of cocyclic maps from $X$ to $K(\mathbb{Z}, m)$. A space $X$ satisfying $G^{m}(X)=H^{m}(X)$ for all $m$ is called a $G^{\prime}$-space. It is

The authors were partially supported by KOSEF891-0103-008-2 and TGRC during this research.

(C) 1995 Australian Mathematical Society 0263-6115/95 \$A2.00+0.00 
known [7] that any co- $H$-space is a $G^{\prime}$-space.

The purpose of this paper is to examine a class of spaces intermediate between $H$-spaces and $G$-spaces and to dualize. In 1987, Aguade introduced and studied $T$ spaces in [2]. A space $X$ is called a $T$-space if the fibration $\Omega X \rightarrow X^{S^{1}} \rightarrow X$ is fiber homotopy equivalent to the trivial fibration $\Omega X \rightarrow X \times \Omega X \rightarrow X$, where $X^{S^{1}}$ is the free loop space of $X$. In section 2, we characterize $T$-spaces by the Gottlieb groups and use them to show that any $H$-space is a $T$-space and any $T$-space is a $G$-space. In 1981, Aguade showed [1] that, in the category of spheres, only $S^{1}, S^{3}$ and $S^{7}$ are $T$-spaces. In fact, we can easily show that $H$-spaces, $T$-spaces and $G$-spaces are equivalent in the category of spheres. Moreover, there are many $T$-spaces which are not $H$-spaces. We show that $X$ is a $T$-space if and only if $X^{S^{1}}$ is a $T$-space when $X$ is a simply-connected space. In Section 3, we introduce a co- $T$-space as a dual space of a $T$-space and characterize co- $T$-spaces by the dual Gottlieb groups, and use it to show that any co- $H$-space is a co- $T$-space and any co- $T$-space is a $G^{\prime}$-space. Moreover, we obtain that for any space $A$, the group $[X, \Omega A]$ is abelian when $X$ is a co-T-space. There is a $G^{\prime}$-space but not a co-T-space. We show that if $X$ is a co- $T$-space, then all cup products vanish in $X$. Most results in Section 2 are dualized. Throughout this paper, space means a space of the homotopy type of a connected locally finite $C W$ complex. We assume also that spaces have non-degenerate base points. All maps shall mean continuous functions. All homotopies and maps are to respect base points (except for the case of the mapping space $X^{A}$ ). The base point as well as the constant map will be denoted by *. For simplicity, we use the same symbol for a map and its homotopy class. Also, we denote by $[X, Y]$ the set of homotopy classes of pointed maps $X \rightarrow Y$. The identity map of space will be denoted by 1 when it is clear from the context. The diagonal map $\Delta: X \rightarrow X \times X$ is given by $\Delta(x)=(x, x)$ for each $x \in X$, the folding map $\nabla: X \vee X \rightarrow X$ is given by $\nabla(x, *)=\nabla(*, x)=x$ for each $x \in X . \Sigma X$ denote the reduced suspension of $X$ and $\Omega X$ denote the based loop space of $X$. The adjoint functor from the group $[\Sigma X, Y]$ to the group $[X, \Omega Y]$ will be denoted by $\tau$. The symbols $e$ and $e^{\prime}$ denote $\tau^{-1}\left(1_{\Omega X}\right)$ and $\tau\left(1_{\Sigma X}\right)$ respectively.

\section{2. $T$-spaces and cyclic maps}

In this section we characterize $T$-spaces by the Gottlieb groups and use them to study the relationships between $H$-spaces, $T$-spaces and $G$-spaces. We also study some properties of $T$-spaces. It is known [13] that if $A$ is a co- $H$-group, then $G(A, X)$ is an abelian subgroup of $[A, X]$. On the other hand, $G(A, X)$ is the evaluation subgroup given by Gottlieb [5,6], when $A$ is a sphere. It is clear that if $f: A \rightarrow X$ is a cyclic map and $\theta: B \rightarrow A$ is an arbitrary map, then $f \theta: B \rightarrow X$ is a cyclic map. It is also easily obtained that an $H$-space may be characterized by the Gottlieb set as 
follows;

PROPOSITION 2.1 [10]. The following are equivalent:

(1) $X$ is an $H$-space;

(2) $1_{X}$ is cyclic;

(3) $G(A, X)=[A, X]$ for any space $A$.

Aguade showed [2] that $X$ is a $T$-space if and only if $X$ is a $T_{1}$-space. In fact, $X$ being a $T_{1}$-space means that $e: \Sigma \Omega X \rightarrow X$ is cyclic. Thus we obtain the next theorem which shows that a $T$-space may be characterized by the Gottlieb group.

THEOREM 2.2. The following are equivalent:

(1) $X$ is a $T$-space;

(2) $e: \Sigma \Omega X \rightarrow X$ is cyclic;

(3) $G(\Sigma A, X)=[\Sigma A, X]$ for any space $A$.

PROOF. The assertion (1) if and only if (2) follows from Proposition 4.1 in [2]. (2) implies (3): Let $f: \Sigma A \rightarrow X$ be a map. Then we have, from the fact that $f=e \Sigma \tau(f): \Sigma A \rightarrow X$ and $e$ is cyclic, that $f: \Sigma A \rightarrow X$ is cyclic. (3) implies (2): Take $A=\Omega X$. Then $e: \Sigma \Omega X \rightarrow X$ is cyclic.

Let $X^{\Sigma A}$ be the space of maps from $\Sigma A$ to $X$ with the compact-open topology. For a based map $f: \Sigma A \rightarrow X$, let $X_{f}^{\Sigma A}$ be the path component of $X^{\Sigma A}$ containing $\mathrm{f}$. Let $\left(X_{f}^{\Sigma A}\right)_{0}$ denote the space of base point preserving maps in $X_{f}^{\Sigma A}$. In general, the components of $X^{\Sigma A}$ almost never have the same homotopy type. However, it is well known that if $X$ is an $H$-space, then $X_{f}^{S^{p}}$ and $X_{g}^{S^{p}}$ have the same homotopy type for arbitrary $\mathrm{f}$ and $\mathrm{g}$ in $\pi_{p}(X)$. Clearly the evaluation map $\omega: X_{f}^{\Sigma A} \rightarrow X$ is a fibration with fiber $\left(X_{f}^{\Sigma A}\right)_{0}$. The second author showed [16] that $f: \Sigma A \rightarrow X$ is cyclic if and only if $X_{f}^{\Sigma A}$ is fiber homotopy equivalent to $X_{*}^{\Sigma A}$. From the above fact and Theorem 2.2 , we can get the following corollary.

COROLLARY 2.3. If $X$ is a $T$-space, then $X_{f}^{\Sigma A}$ and $X_{g}^{\Sigma A}$ have the same homotopy type for arbitrary $f$ and $g$ in $[\Sigma A, X]$.

It is known [2] that if $X$ is a $T$-space, then all Whitehead products vanish in $X$. The following corollary says that a $T$-space has a more powerful property.

COROLLARY 2.4. If $X$ is a $T$-space, then all the generalized Whitehead products vanish in $X$.

ProOF. Suppose $X$ is a $T$-space. Let $f: \Sigma A \rightarrow X$ and $g: \Sigma B \rightarrow X$ be arbitrary maps. From Theorem 2.2, $g: \Sigma B \rightarrow X$ is cyclic. Thus there is a map $G: X \times \Sigma B \rightarrow$ 
$X$ such that $G j=\nabla(1 \vee g)$, where $j: X \vee \Sigma B \rightarrow X \times \Sigma B$ is the inclusion. Let $H=G(f \times 1): \Sigma A \times \Sigma B \rightarrow X$. Then $H j^{\prime}=G(f \times 1) j^{\prime}=G j(f \vee 1)=\nabla(f \vee g)$, where $j^{\prime}: \Sigma A \vee \Sigma B \rightarrow \Sigma A \times \Sigma B$ is the inclusion. This proves the corollary.

A based map $f: A \rightarrow X$ is called weakly cyclic [15] if for any sphere $S^{n}$ and any map $\theta: S^{n} \rightarrow A, f \theta: S^{n} \rightarrow X$ is cyclic. In fact, $f: A \rightarrow X$ is weakly cyclic if and only if $f_{*}\left(\pi_{n}(A)\right) \subset G_{n}(X)$ for all $n$. We showed [15] that any cyclic map is a weakly cyclic map, but the converse does not hold. Also, it is clear that if $f: A \rightarrow X$ is a weakly cyclic map and $\theta: B \rightarrow A$ is an arbitrary map, then $f \theta: B \rightarrow X$ is weakly cyclic.

THEOREM 2.5. The following are equivalent:

(1) $X$ is a $G$-space;

(2) $e: \Sigma \Omega X \rightarrow X$ is weakly cyclic;

(3) $G\left(S^{n}, X\right)=\left[S^{n}, X\right]$ for any sphere $S^{n}$.

ProOF. (1) implies (2): Since $X$ is a $G$-space, the identity map $1_{X}$ of $X$ is weakly cyclic. Thus we have that $e=1_{X} e: \Sigma \Omega X \rightarrow X$ is weakly cyclic. (2) implies (3): Let $f: S^{n} \rightarrow X$ be a map. We may assume that $S^{n}=\Sigma S^{n-1}$. It is clear that if $h: A \rightarrow B$ is a homotopy equivalence, then $h^{*}: G(B, X) \rightarrow G(A, X)$ is a one-to-one correspondence. Since $f=e \Sigma \tau(f): \Sigma S^{n-1} \rightarrow X$ and $e: \Sigma \Omega X \rightarrow X$ is weakly cyclic, we have, from the definition of weakly cyclic, that $f: S^{n} \rightarrow X$ is cyclic. (3) implies (1): This follows from the definition of a $G$-space.

From the above Proposition 2.1, Theorems 2.2 and 2.5, we get the relationships between $H$-spaces, $T$-spaces and $G$-spaces as follows.

COROLlary 2.6. Any $H$-space is a $T$-space and any $T$-space is a $G$-space.

We will obtain many examples of $T$-spaces which are not $H$-spaces from Corollaries 2.13 and 2.14. But it remains open whether or not there are any $G$-spaces that are not $T$-spaces. However, it is known [1] that, in the category of spheres, only $S^{1}, S^{3}$ and $S^{7}$ are $T$-spaces. This result can be easily obtained from the following proposition.

Proposition 2.7. Let $X$ be a co- $H$-space. Then $X$ is an $H$-space if and only if $X$ is a $T$-space.

PROOF. It is sufficient to show that if $X$ is a $T$-space and co- $H$-space, then $X$ is an $H$-space. Since $X$ is a co- $H$-space, there is a map $s: X \rightarrow \Sigma \Omega X$ such that $e s \sim 1_{X}$. Since $e: \Sigma \Omega X \rightarrow X$ is cyclic, $1_{X}$ is cyclic and $X$ is an $H$-space. This proves the proposition. 
COROLLARY 2.8. $H$-spaces, $T$-spaces and $G$-spaces are equivalent in the category of spheres.

Proof. Suppose $S^{n}$ is a $G$-space. Since $\pi_{n}\left(S^{n}\right)=G_{n}\left(S^{n}\right), 1_{S^{n}}$ is cyclic and $S^{n}$ is an $H$-space. Thus we know, from Corollary 2.6, that $H$-spaces, $T$-spaces and $G$-spaces are equivalent in the category of spheres.

Proposition 2.9. Let $X$ be a $T$-space. If there is a map $r: X \rightarrow Y$ such that $r i \sim 1_{Y}$, where $i: Y \rightarrow X$, then $Y$ is a $T$-space.

Proof. Since $e_{X}: \Sigma \Omega X \rightarrow X$ is cyclic, we have that $i e_{Y}=e_{X} \Sigma \Omega i: \Sigma \Omega Y \rightarrow X$ is cyclic. It is known [13] that if $g: X \rightarrow Y$ is a map which has a right homotopy inverse and $f: A \rightarrow X$ is cyclic, then $g f: A \rightarrow Y$ is cyclic. Thus we have that $e_{Y} \sim r\left(i e_{Y}\right): \Sigma \Omega Y \rightarrow Y$ is cyclic.

COROLLARY 2.10. A retract of a $T$-space is a $T$-space.

THEOREM 2.11. $X \times Y$ is a $T$-space if and only if $X$ and $Y$ are $T$-spaces.

Proof. Suppose $X \times Y$ is a $T$-space. Then we have, from Corollary 2.10 , that $X$ and $Y$ are $T$-spaces. On the other hand, let $X$ and $Y$ be $T$-spaces. We show that $G(\Sigma A, X \times Y)=[\Sigma A, X \times Y]$ for any space $A$. Let $f: \Sigma A \rightarrow X \times Y$ be a map. Since $X$ and $Y$ are $T$-spaces, $p_{1} f: \Sigma A \rightarrow X$ and $p_{2} f: \Sigma A \rightarrow Y$ are cyclic maps, where $p_{1}: X \times Y \rightarrow X$ and $p_{2}: X \times Y \rightarrow Y$ are projections. It is known [10] that if $f_{1}: A_{1} \rightarrow X_{1}$ and $f_{2}: A_{2} \rightarrow X_{2}$ are cyclic, then so is $f_{1} \times f_{2}: A_{1} \times A_{2} \rightarrow X_{1} \times X_{2}$. Thus $f=\left(p_{1} f \times p_{2} f\right) \Delta: \Sigma A \rightarrow X \times Y$ is cyclic. This proves the theorem.

THEOREM 2.12. Let $X$ be a simply connected space. Then $X$ is a $T$-space if and only if $X^{s^{1}}$ is a $T$-space.

PROOF. Suppose $X$ is a $T$-space. Since $X$ is simply connected, $\Omega X$ is connected. Thus we know, from the definition of a $T$-space and Theorem 2.11 , that $X^{S^{1}}$ is a connected space and $T$-space. On the other hand, suppose $X^{S^{\prime}}$ is a $T$-space. Consider the inclusion $i: X \rightarrow X^{S^{1}}, i(x)=\hat{x}$, where $\hat{x}(t)=x$ for all $t \in S^{1}$. Then the evaluation map $p: X^{S^{1}} \rightarrow X$ is a retraction of $X^{s^{1}}$ to $X$. Thus we have, from Corollary 2.10 , that $X$ is a $T$-space.

Consider the two stage Postnikov system

$$
K\left(\mathbb{Z}_{2}, 2 n-1\right) \rightarrow \mathbb{E}_{n} \rightarrow K(\mathbb{Z}, 2)
$$


with $k$-invariant $\alpha^{n} \in H^{2 n}\left(K(\mathbb{Z}, 2) ; \mathbb{Z}_{2}\right), n \geq 2$, where $\alpha$ is the generator of $H^{2}\left(K(\mathbb{Z}, 2) ; \mathbb{Z}_{2}\right)$. Then the following facts were known by Haslam (See [7, Theorem 1.2.10]).

(1) $\mathbb{E}_{n}$ is an $H$-space if and only if $n=2^{k}$;

(2) $\mathbb{E}_{n}$ is a $G$-space if and only if $\mathrm{n}$ is even.

COROLLARY 2.13. $\mathbb{E}_{n}^{S^{1}}$ is a $T$-space if and only if $n$ is even.

PROOF. Since $K\left(\mathbb{Z}_{2}, 2 n-1\right) \rightarrow \mathbb{E}_{n} \rightarrow K(\mathbb{Z}, 2)$ is the principal fibration induced by $\alpha^{n}: K(\mathbb{Z}, 2) \rightarrow K\left(\mathbb{Z}_{2}, 2 n\right)$ for $n \geq 2, \mathbb{E}_{n}$ is a simply-connected space. From Theorem 2.12 and Corollary 2.6 , and the above fact (2), it is sufficient to show that if $n$ is even, $\mathbb{E}_{n}$ is a $T$-space. Let $m^{*}: H^{*}\left(K(\mathbb{Z}, 2) ; \mathbb{Z}_{2}\right) \rightarrow H^{*}\left(K(\mathbb{Z}, 2) ; \mathbb{Z}_{2}\right) \otimes H^{*}\left(K(\mathbb{Z}, 2) ; \mathbb{Z}_{2}\right)$ be the homomorphism induced by the multiplication $m: K(\mathbb{Z}, 2) \times K(\mathbb{Z}, 2) \rightarrow K(\mathbb{Z}, 2)$. On the other hand, $K(\mathbb{Z}, 2)$ has a unique $T$-structure $r: K(\mathbb{Z}, 2)^{S^{1}} \rightarrow \Omega K(\mathbb{Z}, 2)$ which comes from the $H$-structure $m$ of $K(\mathbb{Z}, 2)$ (see [2]). Let $\omega: H^{i}\left(K(\mathbb{Z}, 2) ; \mathbb{Z}_{2}\right) \rightarrow$ $H^{i-1}\left(\Omega K(\mathbb{Z}, 2) ; \mathbb{Z}_{2}\right)$ be the suspension and $h: K(\mathbb{Z}, 2) \times \Omega K(\mathbb{Z}, 2) \rightarrow K(\mathbb{Z}, 2)^{S^{1}}$ be a map given by $h(x, \eta)(t)=m(x, \eta(t))$, where $\eta \in \Omega K(\mathbb{Z}, 2)$. Then $(r h)^{*}(y)=1 \otimes y$ for all $y \in H^{*}\left(\Omega K(\mathbb{Z}, 2) ; \mathbb{Z}_{2}\right)$ and $m^{*}\left(\alpha^{n}\right)=\sum_{i=0}^{n} n C i\left(\alpha^{n-i} \otimes \alpha^{i}\right)$, where the binomial coefficient $n C i$ is taken $\bmod 2$. Since $n C 1 \equiv 0 \bmod 2, m^{*}\left(\alpha^{n}\right)=\alpha^{n} \otimes 1+1 \otimes \alpha^{n}+$ $\sum_{i=2}^{n-2} n C i\left(\alpha^{n-i} \otimes \alpha^{i}\right)$. Since $\omega\left(\alpha^{i}\right) \in H^{2 i-1}\left(\Omega K(\mathbb{Z}, 2) ; \mathbb{Z}_{2}\right)=0$ for all $i \geq 2$, we know, from Proposition 2.2 [2], that $\alpha^{n}: K(\mathbb{Z}, 2) \rightarrow K\left(\mathbb{Z}_{2}, 2 n\right)$ is a $T$-map. Thus we know, from Proposition 2.1 [2], that $\mathbb{E}_{n}$ is a $T$-space.

COROLLARY 2.14. $\mathbb{E}_{n}^{S^{1}}$ is an $H$-space if and only if $n=2^{k}$.

ProOF. Let $\mathbb{E}_{n}^{S^{1}}$ be an $H$-space. Then the evaluation map $p: \mathbb{E}_{n}^{S^{1}} \rightarrow \mathbb{E}_{n}$ is a retraction of $\mathbb{E}_{n}^{S^{\prime}}$ to $\mathbb{E}_{n}$. It is well known that a retract of an $H$-space is an $H$-space. Thus we obtain, from the Haslam's result above, that $n=2^{k}$. Suppose $n=2^{k}$. Since $\mathbb{E}_{n}$ is a $T$-space, $\mathbb{E}_{n}^{S^{1}}$ is homotopy equivalent to $\mathbb{E}_{n} \times \Omega \mathbb{E}_{n}$. It is also well-known that if $X$ and $Y$ are $H$-spaces, then $X \times Y$ is an $H$-space. Thus we know that $\mathbb{E}_{n}^{S^{1}}$ is an $H$-space.

Thus we know, in the proof of Corollary 2.13, that $T$-spaces are equivalent to $G$-spaces in the class $\left\{\mathbb{E}_{n} \mid n \geq 2\right\}$ of $\mathbb{E}_{n}$, and $\mathbb{E}_{10}$ is an example of $T$-space which is not an $H$-space. Moreover, we may get many new $G$-spaces $\mathbb{E}_{n}^{S^{1}}$ which are not $H$-spaces.

THEOREM 2.15. Let $Z$ be a homotopy equivalent to $X \times Y$ for some space $Y$. Then $X$ is a T-space if and only if there are maps $r: Z \rightarrow X$ and $i: X \rightarrow Z$ such that $r i \sim 1_{X}$ and ie $: \Sigma \Omega X \rightarrow Z$ is cyclic. 
Proof. Suppose $X$ is a $T$-space. Let $f: Z \rightarrow X \times Y, g: X \times Y \rightarrow Z$ be maps such that $g f \sim 1_{Z}$ and $f g \sim 1_{X \times Y}$. Let $r=p_{1} f: Z \rightarrow X$ and $i=g i_{1}: X \rightarrow Z$, where $p_{1}: X \times Y \rightarrow X$ is the projection and $i_{1}: X \rightarrow X \times Y$ is the inclusion. Then $r i=p_{1} f g i_{1} \sim p_{1} i_{1}=1_{X}$. Now we show that $i e: \Sigma \Omega X \rightarrow Z$ is cyclic. Since $X$ is a $T$-space,there is a map $E: \Sigma \Omega X \times X \rightarrow X$ such that $E j=\nabla(e \vee 1)$. Consider the map $F=g(E \times 1)(1 \times f): \Sigma \Omega X \times Z \rightarrow Z$. Then $F j^{\prime} \sim \nabla\left(i e \vee 1_{Z}\right)$. Thus ie $: \Sigma \Omega X \rightarrow Z$ is cyclic. On the other hand, suppose there is a map $r: Z \rightarrow X$ which has a right homotopy inverse $i: X \rightarrow Z$ and $i e: \Sigma \Omega X \rightarrow Z$ is cyclic. Then we know that $e \sim r(i e): \Sigma \Omega X \rightarrow X$ is cyclic. Thus $X$ is a $T$-space.

\section{3. co- $T$-spaces and cocyclic maps}

In this section we introduce a co- $T$-space which is the dual of a $T$-space, characterize co- $T$-spaces by the dual Gottlieb groups, and use them to study the relationships between co- $H$-spaces, co- $T$-spaces and $G^{\prime}$-spaces. We study some properties of co- $T$-spaces. We also define a weakly cocyclic map, and characterize $G^{\prime}$-spaces by these maps. It is known [12] that if $A$ is an $H$-group, then $D G(X, A)$ is an abelian subgroup of $[X, A]$. On the other hand, $D G(X, A)$ is the coevaluation subgroup given by Haslam [7], when $A$ is a $K(\mathbb{Z}, n)$. It is also known [13] that if $f: X \rightarrow A$ is a cocyclic map and $\theta: A \rightarrow B$ is an arbitrary map, then $\theta f: X \rightarrow B$ is a cocyclic map. It is easily shown that a co- $H$-space may be characterized by the dual Gottlieb set as follows.

PROPOSITION 3.1 [12]. The following are equivalent:

(1) $X$ is a co-H-space;

(2) $1_{X}$ is cocyclic;

(3) $D G(X, A)=[X, A]$ for any space $A$.

DEFINITION 3.2. A space $X$ is called a co-T-space if $e^{\prime}: X \rightarrow \Omega \Sigma X$ is cocyclic. We show that a co- $T$-space may be characterized by the dual Gottlieb group as follows;

THEOREM 3.3. The following are equivalent:

(1) $X$ is a co-T-space;

(2) $e^{\prime}: X \rightarrow \Omega \Sigma X$ is cocyclic;

(3) $D G(X, \Omega A)=[X, \Omega A]$ for any space $A$.

Proof. (1) if and only if (2). This follows from the definition of co-T-space. (2) implies (3). Let $f: X \rightarrow \Omega A$ be a map. From the fact $f=\Omega \tau^{-1}(f) e^{\prime}: X \rightarrow \Omega A$ 
and $e^{\prime}$ is cocyclic, we know that $f: X \rightarrow \Omega A$ is a cocyclic. (3) implies (2). Take $A=\Sigma X$. Then $e^{\prime}: X \rightarrow \Omega \Sigma X$ is cocyclic.

It is known [12] that $D G(X, \Omega A)$ is an abelian subgroup of $[X, \Omega A]$ for any space $A$. Thus we know, from Theorem 3.3, that if $X$ is a co-T-space, then $[X, \Omega A]$ is abelian for any space $A$. Also, $\operatorname{Lim}$ showed [12] that $D G(X, \Omega A) \subset D W(X, \Omega A) \subset$ $[X, \Omega A]$, where $D W(X, \Omega A)=\left\{\alpha \in[X, \Omega A] \mid[\alpha, \beta]^{\prime}=0\right.$ for all $\beta \in[X, \Omega B]$ for all $B\}$, and [, $]^{\prime}$ is the dual generalized Whitehead product [3]. Thus we obtain the following corollary.

COROLLARY 3.4. If $X$ is a co-T-space, then all the dual generalized Whitehead products vanish in $X$.

Also, it is known [9] that $\Sigma X$ is homotopy commutative if and only if $\left[e^{\prime}, e^{\prime}\right]^{\prime}=0$. Thus we know, from Corollary 3.4, that if $X$ is a co- $T$-space, then $\Sigma X$ is homotopy commutative.

EXAMPLE 3.5. Haslam showed [7] that the real projective space $\mathbb{R} P^{2}$ is a $G^{\prime}$-space but not co- $H$-space. In fact, he showed that $D G\left(\mathbb{R} P^{2}, K\left(\mathbb{Z}_{2}, 1\right)\right) \neq\left[\mathbb{R} P^{2}, K\left(\mathbb{Z}_{2}, 1\right)\right]$. Thus we know, from the fact $D G\left(\mathbb{R} P^{2}, \Omega K\left(\mathbb{Z}_{2}, 2\right)\right) \neq\left[\mathbb{R} P^{2}, \Omega K\left(\mathbb{Z}_{2}, 2\right)\right]$ and Theorem 3.3 , that $\mathbb{R} P^{2}$ is not a co- $T$-space.

We can identify $H^{m}(X ; \pi)$ with $[X, K(\pi, m)]$, and define the coevaluation subgroup $G^{m}(X ; \pi)$ of $H^{m}(X ; \pi)$ to be the set of all homotopy classes of cocyclic maps from $X$ to $K(\pi, m)$. The group $G^{m}(X)=G^{m}(X ; \mathbb{Z})$ is the dual to the evaluation subgroup $G_{m}(X)$ of $\pi_{m}(X)$ considered in [6].

COROLlARY 3.6. If $X$ is a co-T -space, then $G^{m}(X ; \pi)=H^{m}(X ; \pi)$ for all $m$ and $\pi$.

PROOF. Let $h: K(\pi, m) \rightarrow \Omega K(\pi, m+1)$ be a homotopy equivalence and let $f: X \rightarrow K(\pi, m)$ be a representative of an element of $H^{m}(X ; \pi)$. Then we have, from Theorem 3.3, that $h f: X \rightarrow \Omega K(\pi, m+1)$ is cocyclic. Since $h: K(\pi, m) \rightarrow$ $\Omega K(\pi, m+1)$ is a homotopy equivalence, $f: X \rightarrow K(\pi, m)$ is cocyclic. This proves the corollary.

Let $R$ be a ring and set $P^{m}(X ; R)=\left\{\alpha \in H^{m}(X ; R) \mid \beta \cup \alpha=0\right.$ for all $\beta \in$ $\left.\tilde{H}^{*}(X ; R)\right\}$, where $\beta \cup \alpha$ denotes the cup product of $\beta$ and $\alpha$. It is known [7] that $G^{m}(X ; R) \subseteq P^{m}(X ; R)$ for all $m$ and $R$. Thus we have the following corollary.

COROLlARY 3.7. If $X$ is a co-T-space, then all cup products vanish in $X$. 
DEFINITION 3.8. A based map $f: X \rightarrow A$ is called weakly cocyclic if for any $n \geq 1$ and any map $\theta: A \rightarrow K(\mathbb{Z}, n), \theta f: X \rightarrow K(\mathbb{Z}, n)$ is cocyclic. In fact $f: X \rightarrow A$ is weakly cocyclic if and only if $f^{*}\left(H^{n}(A)\right) \subset G^{n}(X)$ for all $n \geq 1$. The set of all homotopy classes of weakly cocyclic maps from $X$ to $A$ is denoted by $W D G(X, A)$.

Any cocyclic map is a weakly cocyclic map, but the converse does not hold. It follows from Proposition 3.1 and Example 3.5 that the identity map of $\mathbb{R} P^{2}$ is not cocyclic. But we easily know, from the fact that $\mathbb{R} P^{2}$ is a $G^{\prime}$-space, that the identity map of $\mathbb{R} P^{2}$ is weakly cocyclic. It is clear that if $f: X \rightarrow A$ is an weakly cocyclic map and $\theta: A \rightarrow B$ is an arbitrary map, then $\theta f: X \rightarrow B$ is weakly cocyclic.

LEMMA 3.9. Let $X$ be any space. If $A$ is homotopy equivalent to a $K(\mathbb{Z}, n)$, then $f: X \rightarrow A$ is cocyclic if and only if $f: X \rightarrow A$ is weakly cocyclic.

PROOF. Clearly any cocyclic map is a weakly cocyclic map. Now suppose that $f: X \rightarrow A$ is weakly cocyclic. Since $A$ is homotopy equivalent to $K(\mathbb{Z}, n)$, there exist maps $k: A \rightarrow K(\mathbb{Z}, n)$ and $h: K(\mathbb{Z}, n) \rightarrow A$ such that $h k \sim 1$. Since $f: X \rightarrow A$ is weakly cocyclic, $k f: X \rightarrow K(\mathbb{Z}, n)$ is cocyclic. Thus we have that $f \sim h(k f): X \rightarrow A$ is cocyclic. This proves the lemma.

THEOREM 3.10. The following are equivalent:

(1) $X$ is a $G^{\prime}$-space;

(2) $e^{\prime}: X \rightarrow \Omega \Sigma X$ is weakly cocyclic;

(3) $D G(X, \Omega K(\mathbb{Z}, n+1))=[X, \Omega K(\mathbb{Z}, n+1)]$ for all $n$.

Proof. (1) implies (2): Since $X$ is a $G^{\prime}$-space, the identity map 1 of $X$ is weakly cocyclic. Thus we have that $e^{\prime}=e^{\prime} 1_{X}: X \rightarrow \Omega \Sigma X$ is weakly cocyclic. (2) implies (3): Let $f: X \rightarrow \Omega K(\mathbb{Z}, n+1)$ be a map. Then we have, from the fact that $f=\Omega \tau^{-1}(f) e^{\prime}: X \rightarrow \Omega K(\mathbb{Z}, n+1)$ and $e^{\prime}$ is weakly cocyclic, that $f: X \rightarrow \Omega K(\mathbb{Z}, n+1)$ is weakly cocyclic. Let $h: K(\mathbb{Z}, n) \rightarrow \Omega K(\mathbb{Z}, n+1)$ be a homotopy equivalence. Since $h_{*}: W D G(X, A) \rightarrow W D G(X, B)$ is a one-to-one correspondence, there is a weakly cocyclic map $g: X \rightarrow K(\mathbb{Z}, n)$ such that $h g \sim f$. Thus we have, from Lemma 3.9, that $g: X \rightarrow K(\mathbb{Z}, n)$ is cocyclic. Since $f \sim h g$, $f: X \rightarrow \Omega K(\mathbb{Z}, n+1)$ is cocyclic. (3) implies (1): Since there is a homotopy equivalence $h: K(\mathbb{Z}, n) \rightarrow \Omega K(\mathbb{Z}, n+1), h_{*}:[X, K(\mathbb{Z}, n)] \rightarrow[X, \Omega K(\mathbb{Z}, n+1)]$ is a one-to-one correspondence. Thus it follows from the fact that $h_{*}: D G(X, A) \rightarrow$ $D G(X, B)$ is a one-to-one correspondence and the definition of $G^{\prime}$-space.

From the above Proposition 3.1, Theorems 3.3 and 3.10, we obtain the relationships of co- $H$-spaces, co- $T$-spaces and $G^{\prime}$-spaces as follows. 
COROLLARY 3.11. Any co-H-space is a co-T-space, and any co-T-space is a $G^{\prime}$-space.

It is well-known [13] that if $f: X \rightarrow A$ is cocyclic and $i: Y \rightarrow X$ has a left homotopy inverse, then $f i: Y \rightarrow A$ is cocyclic.

PROPOSITION 3.12. Let $X$ be a co-T-space. If there is a map $r: X \rightarrow Y$ which has a right homotopy inverse $i: Y \rightarrow X$, then $Y$ is a co-T-space.

PROOF. Since $e_{X}^{\prime}: X \rightarrow \Omega \Sigma X$ is cocyclic and $e_{Y}^{\prime} r=(\Omega \Sigma r) e_{X}^{\prime}$, we have that $e_{Y}^{\prime} r: X \rightarrow \Omega \Sigma Y$ is cocyclic. Since $i: Y \rightarrow X$ has a left homotopy inverse $r$, we have $e_{Y}^{\prime} \sim\left(e_{Y}^{\prime} r\right) i: Y \rightarrow \Omega \Sigma Y$ is cocyclic and $Y$ is a co-T -space. This proves the proposition.

COROLLARY 3.13. A retract of a co-T-space is a co- $T$-space.

THEOREM 3.14. $X \vee Y$ is a co-T-space if and only if $X$ and $Y$ are co-T-spaces.

Proof. Suppose $X \vee Y$ is a co- $T$-space. Let $r_{1}=p_{1} j: X \vee Y \rightarrow X$ and $r_{2}=p_{2} j: X \vee Y \rightarrow Y$, where $j: X \vee Y \rightarrow X \times Y$ is the inclusion, $p_{1}: X \times Y \rightarrow X$ and $p_{2}: X \times Y \rightarrow Y$ are natural projections. Then $r_{1} i_{1}=1_{X}$ and $r_{2} i_{2}=1_{Y}$, where $i_{1}(x)=(x, *), i_{2}(y)=(*, y)$. It is known [12] that if $f: X \rightarrow A$ and $g: Y \rightarrow B$ are cocyclic maps, then $f \vee g: X \vee Y \rightarrow A \vee B$ is cocyclic. Thus we have that $X$ and $Y$ are co-T-spaces. On the other hand, suppose that $X$ and $Y$ are co- $T$-spaces. Let $h: \Sigma(X \vee Y) \rightarrow \Sigma X \vee \Sigma Y$ be the natural homeomorphism and $k: \Omega \Sigma X \vee \Omega \Sigma Y \rightarrow \Omega(\Sigma X \vee \Sigma Y)$ be given by $k(\omega, *)=\Omega i_{1}(\omega), k(*, \eta)=\Omega i_{2}(\eta)$, where $i_{1}: \Sigma X \rightarrow \Sigma X \vee \Sigma Y$ is given by $i_{1}(\langle x, t\rangle)=(\langle x, t\rangle, *)$ and $i_{2}: \Sigma Y \rightarrow$ $\Sigma X \vee \Sigma Y$ is given by $i_{2}(\langle y, t\rangle)=(*,\langle y, t\rangle)$. Then we have the following commutative diagram

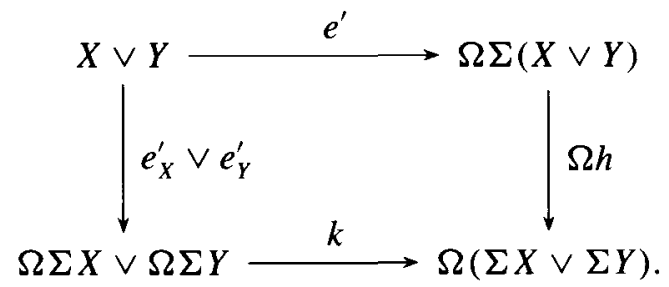

Since $e_{X}^{\prime} \vee e_{Y}^{\prime}$ is cocyclic, $\Omega h e^{\prime}=k\left(e_{X}^{\prime} \vee e_{Y}^{\prime}\right)$ is cocyclic. Since $\Omega h$ is homeomorphism, $e^{\prime}: X \vee Y \rightarrow \Omega \Sigma(X \vee Y)$ is cocyclic. This proves the theorem. 


\section{Acknowledgement}

We would like to thank the referee for helpful suggestions.

\section{References}

[1] J. Aguade, 'On the space of free loops of an odd sphere', Publ. Mat. UAB 25 (1981), 87-90.

[2] — 'Decomposable free loop spaces', Canad. J. Math. 39 (1987), 938-955.

[3] M. Arkowitz, 'The generalized Whitehead product', Pacific J. Math. 12 (1962), 7-23.

[4] G. Dula and D. H. Gottlieb, 'Splitting off H-spaces and Conner-Raymond Splitting theorem', $J$ Fac. Sci. Univ. Tokyo Sect. IA, Math. 37 (1990), 321-334.

[5] D. H. Gottlieb, 'A certain subgroup of the fundamental group', Amer. J. Math. 87 (1965), 840-856.

[6] _ 'Evaluation subgroups of homotopy groups', Amer. J. Math. 91 (1969), 729-756.

[7] H. B. Haslam, $G$-spaces and $H$-spaces (Ph.D. Thesis, University of California, Irvine, 1969).

[8] P. J. Hilton, Homotopy theory and duality (Gordon and Breach, New York, 1965).

[9] C. S. Hoo, 'On the criterion of Stasheff', Canad. J. Math. 21 (1969), 250-255.

[10] K. L. Lim, 'On cyclic maps', J. Austral. Math. Soc., (Series A) 32 (1982), 349-357.

[11] _ ' On evaluation subgroups of generalized homotopy groups', Canad. Math. Bull. 27 (1984), $78-86$.

[12] —_, 'Cocyclic maps and coevaluation subgroups', Canad. Math. Bull. 30 (1987), 63-71.

[13] K. Varadarajan, 'Generalized Gottlieb groups', J. Indian. Math. Soc. 33 (1969), 141-164.

[14] G. W. Whitehead, Elements of homotopy theory, Graduate Texts in Mathematics 61 (Springer, Berlin, 1978).

[15] M. H. Woo and Y. S. Yoon, 'On some properties of G-spaces', Comm. Korean Math. Soc. 2 (1987), 117-121.

[16] Y. S. Yoon, 'On n-cyclic maps', J. Korean Math. Soc. 26 (1989), 17-25.

Department of Mathematics Education

Korea University

136-075, Seoul

Korea
Department of Mathematics

Hannam University 300-791, Taejon

Korea 